\title{
HOLOMORPHIC DISCS WITH DENSE IMAGES
}

\author{
FrAnC FORSTNERIČ AND JÖRG WINKELMANN
}

\begin{abstract}
Let $\Delta$ be the open unit disc in $\mathbb{C}, X$ a connected complex manifold and $\mathcal{D}$ the set of all holomorphic maps $f: \Delta \rightarrow X$ with $\overline{f(\Delta)}=X$. We prove that $\mathcal{D}$ is dense in $\operatorname{Hol}(\Delta, X)$.
\end{abstract}

\section{Introduction}

Let $\Delta_{r}=\{z \in \mathbb{C}:|z|<r\}$ and $\Delta=\Delta_{1}$. In [8] the second author proved that for any irreducible complex space $X$ there exists a holomorphic map $\Delta \rightarrow X$ with dense image, and he raised the question whether the set of all holomorphic maps $\Delta \rightarrow X$ with dense image forms a dense subset of the set $\operatorname{Hol}(\Delta, X)$ of all holomorphic maps $\Delta \rightarrow X$ with respect to the topology of locally uniform convergence.

In this paper we show that the answer to this question is positive if $X$ is smooth, but negative for some singular space.

Theorem 1. For any connected complex manifold $X$ the set of holomorphic maps $\Delta \rightarrow X$ with dense images forms a dense subset in $\operatorname{Hol}(\Delta, X)$. The conclusion fails for some singular complex surface $X$.

The situation is quite different for proper discs, i.e., proper holomorphic maps $\Delta \rightarrow X$. The paper [4] contains an example of a non-pseudoconvex bounded domain $X \subset \mathbb{C}^{2}$ such that a certain nonempty open subset $U \subset X$ is not intersected by the image of any proper holomorphic disc $\Delta \rightarrow X$. On the other hand, proper holomorphic discs exist in great abundance in Stein manifolds [6], [1], [2] and, more generally, in $q$-complete manifolds $X$ for $1 \leq q<\operatorname{dim} X$ [3].

\section{Preparations}

Lemma 1. Let $W_{n}$ be a decreasing sequence (i.e., $W_{n+1} \subset W_{n}$ ) of open sets with $\Delta \subset W_{n} \subset \Delta_{2}$ for every $n$. Let $K=\cap_{n} \bar{W}_{n}$ and assume that the interior of $K$ coincides with $\Delta$. Furthermore assume that there are biholomorphic maps $\phi_{n}: \Delta \rightarrow W_{n}$ with $\phi_{n}(0)=0$ for $n=1,2, \ldots$

Received October 23, 2004.

2000 Mathematics Subject Classification. 32H02.

Key words and phrases. Holomorphic discs, complex spaces.

Research of the first author was supported by grants P1-0291 and J1-6173, Republic of Slovenia. 
Then there exists an automorphism $\alpha \in \operatorname{Aut}(\Delta)$ and a subsequence $\left(\phi_{n_{k}}\right)$ of the sequence $\left(\phi_{n}\right)$ such that $\phi_{n_{k}} \circ \alpha^{-1}$ converges locally uniformly to the identity map $i d_{\Delta}$ on $\Delta$.

Proof. Montel's theorem shows that, after passing to a suitable subsequence, we have $\lim _{n \rightarrow \infty} \phi_{n}=\alpha: \Delta \rightarrow K$ and $\lim _{n \rightarrow \infty}\left(\left.\phi_{n}^{-1}\right|_{\Delta}\right)=\beta: \Delta \rightarrow \bar{\Delta}$. Since the limit maps are holomorphic and satisfy $\alpha(0)=0$ and $\beta(0)=0$, we conclude that $\alpha(\Delta) \subset \operatorname{Int} K=\Delta$ and $\beta(\Delta) \subset \Delta$. Moreover $\alpha \circ \beta=i d_{\Delta}=\beta \circ \alpha$, and hence both $\alpha$ and $\beta$ are automorphisms of $\Delta$ (indeed, rotations $z \rightarrow z e^{i t}$ ).

We also need the following special case of a result of the first author (theorem 3.2 in [5]):

Proposition 1. Let $X$ be a complex manifold, $0<r<1$, $E$ the real line segment $[1,2] \subset \mathbb{C}, K=\bar{\Delta} \cup E, U$ an open neighbourhood of $\bar{\Delta}$ in $\mathbb{C}, S$ a finite subset of $K$ and $f: U \cup E \rightarrow X$ a continuous map which is holomorphic on $U$.

Then there is a sequence of pair of open neighbourhoods $W_{n} \subset \mathbb{C}$ of $K$ and holomorphic maps $g_{n}: W_{n} \rightarrow X$ such that:

1. $\left.g_{n}\right|_{K}$ converges uniformly to $\left.f\right|_{K}$ as $n \rightarrow \infty$, and

2. $g_{n}(a)=f(a)$ for all $a \in S$ and $n \in \mathbb{N}$.

\section{Towards the main result}

In this section we prove the following proposition which is the main technical result in the paper. The first statement in theorem $1(\S 1)$ is an immediate corollary.

Proposition 2. Let $X$ be a connected complex manifold endowed with a complete Riemannian metric and induced distance $d, S$ a countable subset of $X$, $f: \Delta \rightarrow X$ a holomorphic map, $\epsilon>0$ and $0<r<1$.

Then there exists a holomorphic map $F: \Delta \rightarrow X$ such that

(a) $S \subset F(\Delta)$, and

(b) $d(f(z), F(z)) \leq \epsilon$ for all $z \in \Delta_{r}$.

Proof. Let $s_{1}, s_{2}, s_{3}, .$. be an enumeration of the elements of $S$. We shall inductively construct a sequence of holomorphic maps $f_{n}: \Delta \rightarrow X$, numbers $r_{n} \in(0,1)$ and points $a_{1, n}, \ldots, a_{n, n} \in \Delta$ satisfying the following properties for $n=0,1,2, \ldots$ :

1. $f_{0}=f$ and $r_{0}=r$,

2. $\left(r_{n}+1\right) / 2<r_{n+1}<1$,

3. $f_{n}\left(a_{j, n}\right)=s_{j}$ for $n \geq 1$ and $j=1,2, \ldots, n$,

4. $d\left(f_{n}(z), f_{n+1}(z)\right)<2^{-(n+1)} \epsilon$ for all $z \in \Delta_{r_{n}}$, and

5. $d_{\Delta}\left(a_{j, n}, a_{j, n+1}\right)<2^{-n}$ for $j=1,2, \ldots, n$ where $d_{\Delta}$ denotes the Poincaré distance on the unit disc.

Assume inductively that the data for level $n$ (i.e., $f_{n}, r_{n}, a_{j, n}$ ) have been chosen. (For $n=0$ we do not have any points $a_{j, 0}$.) With $n$ fixed we choose an increasing sequence of real numbers $\lambda_{k}$ with $\lambda_{k}>r_{n}$ and $\lim _{k \rightarrow \infty} \lambda_{k}=1$. 
For every $k \in \mathbb{N}$ the map $\widetilde{g}_{k}(z) \stackrel{\text { def }}{=} f_{n}\left(\lambda_{k} z\right) \in X$ is defined and holomorphic on the disc $\Delta_{1 / \lambda_{k}} \supset \bar{\Delta}$. After a slight shrinking of its domain we can extend it continuously to the segment $E=[1,2] \subset \mathbb{C}$ such that the right end point 2 of $E$ is mapped to the next point $s_{n+1} \in S$ (this is possible since $X$ is connected).

Applying proposition 1 to the extended map $\widetilde{g}_{k}$ we obtain for every $k \in \mathbb{N}$ an open neighbourhood $V_{k} \subset \mathbb{C}$ of $K=\bar{\Delta} \cup E$ and a holomorphic map $g_{k}: V_{k} \rightarrow X$ such that

(i) $\left|g_{k}(z)-f_{n}\left(\lambda_{k} z\right)\right|<2^{-k}$ for all $z \in \bar{\Delta}$,

(ii) $g_{k}(2)=s_{n+1}$, and

(iii) $g_{k}\left(a_{j, n} / \lambda_{k}\right)=f_{n}\left(a_{j, n}\right)=s_{j}$ for $j=1, \ldots, n$.

Next we choose a decreasing sequence of simply connected open sets $W_{k} \subset \mathbb{C}$ $(k \in \mathbb{N})$ with $K \subset W_{k} \subset V_{k}$ and $K=\cap_{k} \bar{W}_{k}$. Notice that $\operatorname{Int} K=\Delta$. By lemma 1 there is a sequence of biholomorphic maps $\phi_{k}: \Delta \rightarrow W_{k}$ with $\lim _{k \rightarrow \infty} \phi_{k}=i d_{\Delta}$.

Consider the holomorphic maps $h_{k}=g_{k} \circ \phi_{k}: \Delta \rightarrow X$. By our construction we know that $\lim _{k \rightarrow \infty} h_{k}=f_{n}$ locally uniformly on $\Delta$.

To fulfill the inductive step it thus suffices to choose $f_{n+1}=h_{k}$ for a sufficiently large $k, a_{j, n+1}=a_{j, n} / \lambda_{k}(j=1, \ldots, n), a_{n+1, n+1}=\phi_{k}^{-1}(2)$. Finally we choose a number $r_{n+1}$ satisfying

$$
\max \left\{\left|a_{n+1, n+1}\right|, \frac{r_{n}+1}{2}\right\}<r_{n+1}<1 .
$$

This completes the inductive step.

By properties (2) and (4) the sequence $f_{n}$ converges locally uniformly in $\Delta$ to a holomorphic map $F: \Delta \rightarrow X$. Aided by property (1) we also control $d(f(z), F(z))$ for $z \in \Delta_{r}$. Since the Poincaré metric is complete, property (5) insures that for every fixed $j \in \mathbb{N}$ the sequence $a_{j, n} \in \Delta(n=j, j+1, \ldots)$ has an accumulation point $b_{j}$ inside of $\Delta$, and (3) implies $F\left(b_{j}\right)=s_{j}$ for $j=1,2, \ldots$ Hence $S \subset F(\Delta)$.

\section{Singular spaces}

We use an example of Kaliman and Zaidenberg [7] to show that for a complex spaces $X$ with singularities the set of maps $\Delta \rightarrow X$ with dense image need not be dense in $\operatorname{Hol}(\Delta, X)$. We denote by $\operatorname{Sing}(X)$ the singular locus of $X$.

Proposition 3. There is a singular compact complex surface $S$, a non-constant holomorphic map $f: \Delta \rightarrow S$ and an open neighbourhood $\Omega$ of $f$ in $\operatorname{Hol}(\Delta, S)$ such that $g(\Delta) \subset \operatorname{Sing}(S)$ for every $g \in \Omega$.

Proof. In [7] Kaliman and Zaidenberg constructed an example of a singular surface $S$ with normalization $\pi: Z \rightarrow S$ such that $S$ contains a rational curve $C \simeq \mathbb{P}^{1}$ while $Z$ is smooth and hyperbolic. Denote by $d_{Z}$ the Kobayashi distance function on $Z$. We choose two distinct points $p, q \in C$ and open relatively compact neighbourhoods $V$ of $p$ and $W$ of $q$ in $S$ such that $\bar{V} \cap \bar{W}=\emptyset$. The 
preimages $\pi^{-1}(\bar{V})$ and $\pi^{-1}(\bar{W})$ in $Z$ are also compact, and since $Z$ is hyperbolic we have

$$
r=\min \left\{d_{Z}(x, y): x \in \pi^{-1}(\bar{V}), y \in \pi^{-1}(\bar{W})\right\}>0 .
$$

Fix a point $a \in \Delta$ with $0<d_{\Delta}(0, a)<r$ and let $\Omega$ consist of all holomorphic maps $g: \Delta \rightarrow S$ satisfying $g(0) \in V$ and $g(a) \in W$. Since both $p$ and $q$ are lying on the rational curve $C$, there is a holomorphic map $g: \Delta \rightarrow C$ with $g(0)=p \in V$ and $g(a)=q \in W$; hence the set $\Omega$ is not empty. Clearly $\Omega$ is open in $\operatorname{Hol}(\Delta, S)$.

To conclude the proof it remains to show that $g(\Delta) \subset \operatorname{Sing}(S)$ for all $g \in$ $\Omega$. Indeed, a holomorphic map $g: \Delta \rightarrow S$ with $g(\Delta) \not \subset \operatorname{Sing}(S)$ admits a holomorphic lifting $\widetilde{g}: \Delta \rightarrow Z$ with $\pi \circ \widetilde{g}=g$. If $g \in \Omega$ then by construction

$$
d_{Z}(\widetilde{g}(0), \widetilde{g}(a)) \geq r>d_{\Delta}(0, a)
$$

which violates the distance decreasing property for the Kobayashi pseudometric. This contradiction establishes the claim.

In particular, we see that in this example the set of all holomorphic maps $f: \Delta \rightarrow S$ with dense image does not constitute a dense subset of $\operatorname{Hol}(\Omega, S)$.

\section{References}

[1] B. Drinovec-Drnovšek, Discs in Stein manifolds containing given discrete sets, Math. Z., 239 (2002), 683-702.

[2] - Proper discs in Stein manifolds avoiding complete pluripolar sets, Math. Res. Lett., 11 (2004), 575-581.

[3] - Proper discs in q-convex manifolds, Preprint, March 2005. http://arXiv.org/abs/math/0503449

[4] F. Forstnerič, J. Globevnik, Discs in pseudoconvex domains, Comment. Math. Helv., 67 (1992), 129-145.

[5] F. Forstnerič, Holomorphic Submersions from Stein Manifolds, Ann. Inst. Fourier, 54 (2004), 1913-1942.

[6] J. Globevnik, Discs in Stein manifolds, Indiana Univ. Math. J., 49 (2000), 553-574.

[7] S. Kaliman, M. Zaidenberg, Non-hyperbolic complex space with a hyperbolic normalization, Proc. Amer. Math. Soc., 129 (2001), 1391-1393.

[8] J. Winkelmann, Non-degenerate Maps and Sets, Math. Z., to appear. Online access: http://www.springerlink.com/index/10.1007/s00209-004-0732-2

Institut OF MATHEMATICS, PHYSICS AND MECHANICS, UNIVERSITY OF LJUBLJANA, JADRANSKa 19, 1000 Ljubluana, Slovenia

E-mail address: franc.forstneric@fmf.uni-lj.si

Institut Elie Cartan (Mathématiques), Université Henri Poincaré Nancy 1, B.P. 239, F-54506 Vandegurre-Les-Nancy Cedex, France

E-mail address: jwinkel@member.ams.org

Webpage: http://www.math.unibas.ch/ ${ }^{\sim}$ winkel/ 